\title{
Cubification and Animation of Artistic Shapes
}

\author{
Quentin Corker-Marin \\ The National Centre for Computer \\ Animation \\ Bournemouth University, UK \\ i7624405@bournemouth.ac.uk
}

\author{
Valery Adzhiev \\ The National Centre for Computer \\ Animation \\ Bournemouth University, UK \\ vadzhiev@bournemouth.ac.uk
}

\author{
Alexander Pasko \\ The National Centre for Computer \\ Animation \\ Bournemouth University, UK \\ apasko@bournemouth.ac.uk
}
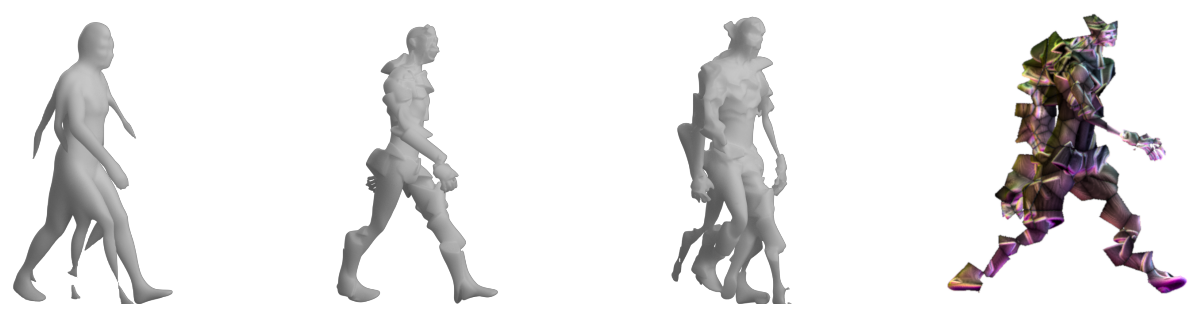

Figure 1: From left to right: 4D Cubist Camera projection of articulated human figure with space-time blending; Cubification of articulated human figure; Cubification of articulated human figure with 4D Cubist camera projection; 3D Cubist camera projection of cubified figure.

\begin{abstract}
This poster describes an original approach to creating static and dynamic sculptures in a cubist style. We propose a novel method for faceting and local distortion thus adding cubist features and generating time-variant sculptural shapes. We introduce the concept of a $4 \mathrm{D}$ cubist camera for blending multiple projections from $4 \mathrm{D}$ spacetime to 3D space. We describe a practical pipeline embracing all the main phases of production of static and dynamic cubist shapes. The proposed techniques are implemented and experimental results are presented.
\end{abstract}

\section{CCS CONCEPTS}

- Computing methodologies $\rightarrow$ Computer graphics; Procedural animation; Shape analysis;

\section{KEYWORDS}

Hybrid Representation, Space-Time Blending, Cubism, 3D Printing

ACM Reference format:

Quentin Corker-Marin, Valery Adzhiev, and Alexander Pasko. 2017. Cubification and Animation of Artistic Shapes. In Proceedings of SCA '17, Los Angeles, CA, USA, July 28-30, 2017, 3 pages.

https://doi.org/10.1145/3099564.3108162

\section{INTRODUCTION}

Dynamic sculptures created with help of computer-aided design and animation tools and related to a proper artistic style and tradition within a particular artistic movement are surprisingly rare. Of

Permission to make digital or hard copies of part or all of this work for personal or classroom use is granted without fee provided that copies are not made or distributed for profit or commercial advantage and that copies bear this notice and the full citation on the first page. Copyrights for third-party components of this work must be honored.

For all other uses, contact the owner/author(s).

SCA '17, July 28-30, 2017, Los Angeles, CA, USA

(c) 2017 Copyright held by the owner/author(s)

ACM ISBN 978-1-4503-5091-4/17/07 .\$15.00

https://doi.org/10.1145/3099564.3108162 course, there are well-established methods and tools allowing for modelling, rendering and animation of an almost infinite variety of shapes. There are also some powerful design tools for sculpturing static shapes [Séquin 2005]. However, creating dynamic art shapes are mostly concerned with traditional engineering and manufacturing practices. In this project we consider an original approach to creating artistic shapes related to the cubism movement.

This project has also served as inspiration for some original theoretical concepts integrating modelling and animation in the context of multidimensional conceptual space. There are parallels between cubism and $4 \mathrm{D}$ space, $\mathrm{nD}$ space and non-Euclidian geometries [Robbin 2006] which have influenced the project.

\section{OUR APPROACH}

\subsection{Overview}

Cubist painters moved away from traditional methods of projecting a $3 \mathrm{D}$ scene onto a $2 \mathrm{D}$ canvas, instead experimenting with using multiple viewpoints in a single composition. We propose to look at the theme of reducing the dimensionality of a scene through the new concept of a 4D cubist camera, which, by analogy with the well-established 3D cubist camera [Glassner 2004], is projecting two or more instances of a time-variant shape from $4 \mathrm{D}$ space-time to $3 \mathrm{D}$ space with possible preliminary blending between these instances. Whilst the 3D cubist camera [Arpa et al. 2012] deals with just rendering, this novel 4D cubist camera realises integration of modelling and animation phases through blending (i.e. compressing) a few frames generated on the basis of a faceted model with attached time-variant transformations into one thus producing cubist effect.

We propose a general shape model and develop algorithms to implement all the distinctive features of cubist sculptures. Then, combine the tools into a single system with artist's interactive or procedural control. We will use a hybrid representation combining polygonal meshes with signed distance fields (SDFs). We will cover 


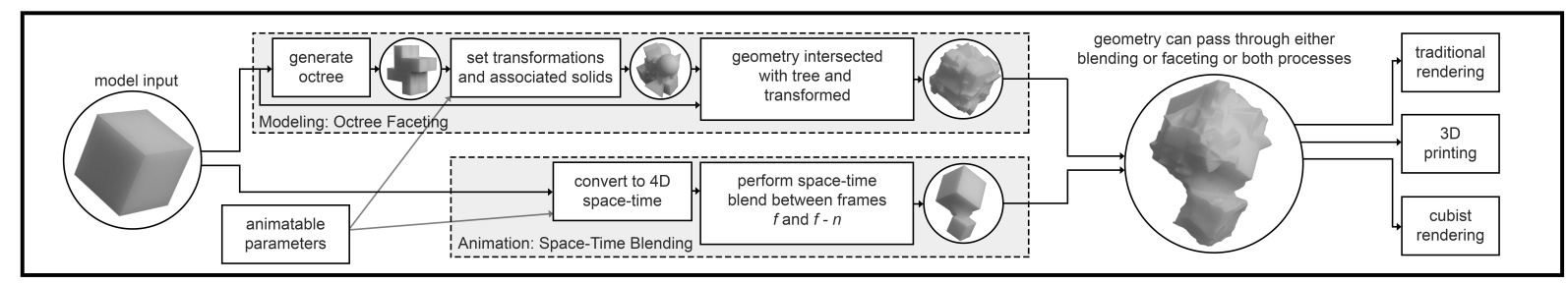

Figure 2: Cubification and animation production pipeline.

all the steps through the full production pipeline from data acquisition, to shape modelling, animation, rendering and 3D printing (Fig. 2).

\subsection{Shape Faceting}
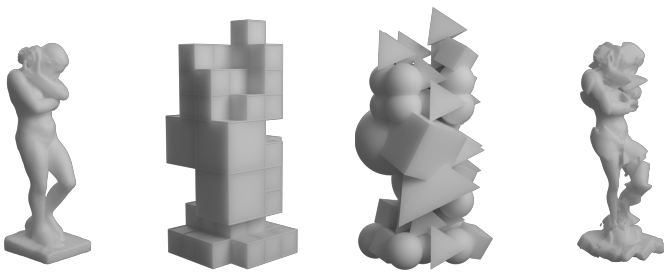

Figure 3: Steps of faceting the given sculpture from left to right: initial shape (scanned model, Three D Scans); corresponding octree; associated primitive solids; rotated and scaled facets combined with manipulated initial mesh.

Cubification of an artistic 3D shape is based on subdivision into solid pieces (facets). Octree-based subdivision is used as it provides a loose form of feature sensitivity. Shapes and transformations are associated with each of the leaf nodes in the resulting tree structure. Each of the shapes are then intersected with the original shape and transformed using their associated transformation. The resulting facets are then combined with the initial mesh to produce the final result (Fig. 3). Facet transformations can be animated by the artist to produce dynamic sculptures.

\subsection{D Cubist Camera}
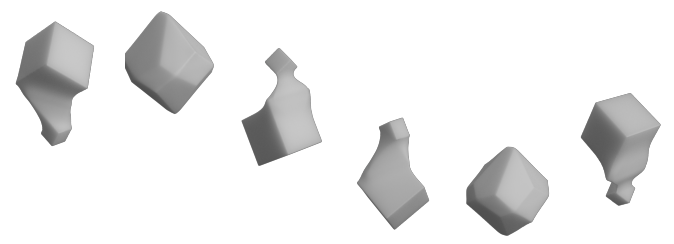

Figure 4: Space-time blending applied to non-adjacent instances of a rotating and oscillating cube.

We employ space-time blending [Pasko et al. 2004] for combining (or compressing) several shape instances (frames) into a single shape. Note that in this process animation is considered as modelling in $4 \mathrm{D}$ with projections to 3D. Space-time blending was used to generate motion trails to follow the facets produced by the octree method described earlier. To achieve this, the blend is performed between non-adjacent shape instances (frames) $t$ and $t-n$. This allows for the effects of the surrounding frames of an animation to influence the current frame being viewed, producing a cubist-type projection of $4 \mathrm{D}$ space-time onto 3D space (see fig. 1 and fig. 4).

\subsection{Implementation and Experiments}

Houdini was a natural choice as the host application for the tools developed in this work. The implemented techniques rely heavily on the use of sparse volumes (discretised SDFs) from the OpenVDB library developed by DreamWorks Animation.
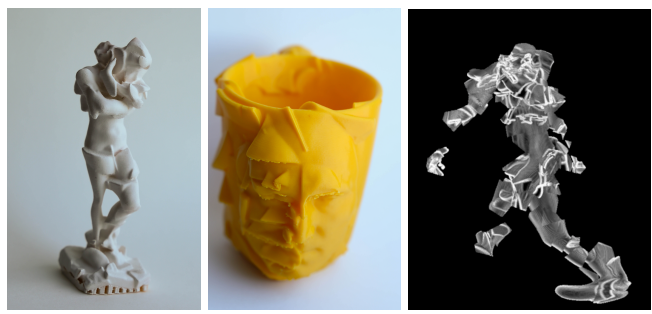

Figure 5: Left to right: $3 D$ printed results showing faceted statue; a faceted mug from 3D scan data; a faceted articulated human rendered with a $3 D$ cubist camera.

\section{CONCLUSION}

We have proposed an original approach to adaptive cubification of existing sculptures including faceting and local distortions. A new concept of a 4D cubist camera as a projection method from 4D to 3D was introduced. A pipeline embracing all the phases of artistic production has been realised. Some artefacts produced during this project can be seen in Fig. 5, the supplementary video and images.

\section{REFERENCES}

Sami Arpa, Abdullah Bulbul, Tolga Capin, and Bulent Ozguc. 2012. Perceptual 3D rendering based on principles of analytical cubism. Computers \& Graphics 36, 8 (2012), 991-1004.

Andrew Glassner. 2004. Digital cubism. IEEE Computer Graphics and Applications 24, 3 (2004), 82-90.

Galina Pasko, Alexander Pasko, and Tosiyasu Kunii. 2004. Space-time blending. Computer Animation and Virtual Worlds 15, 2 (2004), 109-121.

Tony Robbin. 2006. Shadows of Reality: The Fourth Dimension in Relativity, Cubism, and Modern Thought. Yale University Press, New Haven and London.

Carlo H Séquin. 2005. CAD tools for aesthetic engineering. Computer-Aided Design 37, 7 (2005), 737-750 